\title{
Stress effects on gastrointestinal transit in the rat
}

\author{
P ENCK, V MERLIN, J F ERCKENBRECHT, AND M WIENBECK \\ From the Department of Internal Medicine, Division of Gastroenterology, University Hospitals, Dusseldorf, \\ Fed Rep Germany
}

SUmmary Previous investigations of stress effects on gastric emptying, orocaecal, and colonic transit in rats have produced conflicting results. Here one type of stressor, a 'passive avoidance' situation, was used to investigate its effects on gastric emptying, orocaecal and colonic transit. After the rats had been trained to eat a standard amount of semisolid food, gastric emptying was determined $(n=12)$ by the food remaining in the stomach after various periods of rest, or stress exposure. Orocaecal transit $(n=14)$ was determined by breath hydrogen measurements after the food had been labelled with $1 \mathrm{~g}$ lactose. Colonic transit $(n=18)$ was measured as the arrival time of coloured faeces after infusion of a carmine red solution into the caecum through a chronically implanted catheter. The stressor had differential effects on transit through the stomach, small bowel and colon: gastric emptying was delayed $(p<0.05)$ after stress $\left(t \frac{1}{2}=2.66 \mathrm{~h}\right.$ after stress, $1.97 \mathrm{~h}$ at rest). Orocaecal transit was accelerated $(p<0 \cdot 05)$ after stress; transit time decreased from $124 \cdot 3 \mathrm{~min}$ at rest to $86 \cdot 2 \mathrm{~min}$ after stress. Colonic transit was accelerated $(\mathrm{p}<0 \cdot 01)$ under stress, from $15 \cdot 5 \mathrm{~h}$ to $1.29 \mathrm{~h}$. It is concluded that gastrointestinal transit in different parts of the gastrointestinal tract is differently affected by central nervous stimuli.

Investigations of stress effects on gastrointestinal transit in animals have produced conflicting results: an acceleration as well as a delay of transit after stress have been reported for stomach emptying, orocaecal transit, and colonic transit. ${ }^{1-12}$

Several reasons may account for this: (1) The stress models used confound psychological components such as fear, unpredictability, or uncontrollability as a result of restraint, ${ }^{14}$ open field, ${ }^{\circ}{ }^{7}$ noise,,$"$ or light, with physical stress components such as a change in body temperature by cold" 11 or heat, ${ }^{12}$ restricted respiration by wrapping, " physical pain,,$^{23}$ or systemic arousal by motor activity. (2) Most of the measurement techniques require multiple handling of the animals before exposure to the experimental stressor - for example, for oral gavage of the marker. ${ }^{1+5}$ Handling, however, is known to act as a stressor itself, causing sustained raises of plasma catecholamines and cortisol. ${ }^{13}$ (3) Transit studies in

Address for correspondence: Paul Enck. PhD, University Hospitals. Department of Internal Medicine (D). Division of Gastroenterology. Moorenstrasse 5. D-4(K) Dusseldorf 1. Fed Rep Germany.

Accepted for publication 29 September 1988 the upper gut are usually performed with colour or radio-isotope labelled markers in liquid carrier solutions for oral infusion ${ }^{1+5}$ or application through a chronic fistula. "." Gastric emptying of liquids, however, is known to be quite different from that of solid food. ${ }^{1+}$ (4) Transit studies usually do not allow multiple measurements in individual animals as propulsion of the marker within the gut can only be controlled after the animal has been killed. ${ }^{1-5} y_{-12}$

To avoid some of these disadvantages we developed a novel stress model closely resembling a standard 'passive avoidance' situation ${ }^{15}$ and applied it to the study of gastric emptying of solid food (experiment 1), orocaecal transit (experiment 2 ), and to the study of colonic transit (experiment 3 ) in laboratory rats. The experiments were approved by Der Regierungspräsident Düsseldorf under the German animal protection law (Tierschutzgesetz) from 24 July 1972 .

\section{Methods}

EXPERIMENT 1

Twelve female Wistar rats (mean weight: $196.7 \mathrm{~g}$ ) 
were housed individually. Eight days before the test (day 8) the animals were placed on a restricted feeding schedule allowing free access to $25 \mathrm{~g}$ of dry food (Altomin) mixed with water 1:1 for two hours at day 7, for one hour at days 6 to 4 , and for 30 minutes at days 3 to 1 . Feeding time was always 900 am. The animals were fed individually in their home cages at days 8 to 5 and in the test box at days 4 to 1 . During the pretraining and training period the rats received water ad lib. The animals were controlled daily for body weight.

The rats were randomly assigned to two groups (rest or stress) before the test. At test day all rats received $5 \mathrm{~g}$ of the same food for five minutes in the box. The stressor was applied by flooding the plexiglass boxes $(2500 \mathrm{cc})$ with $500 \mathrm{ml}$ water at $30^{\circ} \mathrm{C}$; under control condition (rest) the boxes remained without water. The rats were allowed to climb onto a glass block $(5 \times 4 \times 3 \mathrm{~cm})$ to avoid contact with the water.

In randomised order the rats were removed from their boxes and killed pairwise after 30, 60, 90, 120, 150 , or 180 minutes of stress or rest by an overdose of ether.

The stomach was removed, weighted, cleaned from contents, and weighted again. Weight differences of gastric contents were expressed as \% of actual uptake of food.

In each rat emptying of the stomach was expressed as $\%$ of remaining food previously ingested. A bivariate distribution (stomach content $v$ time) was plotted and linear and non-linear regression analyses were performed (BMDP $3 R$ on an IBM PC AT) to determine the curve which best fits the data - that is, which maximally explains the total data variance (SD) by minimising the residual mean square (RMSQ). Half-emptying of the stomach $\left(t^{1} / 2\right)$ was calculated based on this function. ${ }^{16}$ Differences between stress and rest were tested by the Wilcoxon's signed-ranks test for statistically significant differences. A level of $\mathrm{p}<0.05$ was considered to be significant.

EXPERIMENT 2

Fourteen female Wistar rats (mean body weight: $196 \mathrm{~g}$ ) were placed on a rigid feeding schedule similar to experiment 1 for eight days.

At test day the animals received $15 \mathrm{~g}$ dry food mixed with water (1:1) and with $1 \mathrm{~g}$ lactose at 900 am for 30 minutes in their cages. Immediately after food removal the animals were placed into the test box described above. According to a random plan half of the boxes were then flooded with $500 \mathrm{ml}$ water at $30^{\circ} \mathrm{C}$ while the other half of served as controls without water. Again, rats in a stress box could avoid water contact by climbing onto a glass block. All animals remained under these conditions for three hours. Two days later the procedure was repeated in a crossover fashion.

For measuring of breath hydrogen ${ }^{17}$ the test box was safely closed using a rubber ring between the box and the top. At both ends there were opening of $1 \mathrm{~cm}$ in diameter. One of these openings was connected to a vacuum pump, which continuously exchanges the air within the box while the other hole remained open.

For sampling of cumulative exhaled air, the pump was disconnected by a stop-cock while the other opening was closed by a rubber valve. Sampling was done every 15 minutes for five minutes under rest conditions and for four minutes in stress exposed animals considering a $20 \%$ reduction of the box's air volume by the water used to flood the box.

Samples of $20 \mathrm{ml}$ air were taken from each box by a syringe needle through the valve. Immediately after the samples had been taken, all air within the box was exchanged by the vacuum pump. Control samples were taken occasionally at the end of this period to control whether the pump had removed all hydrogen from within the box.

Hydrogen concentration of the $20 \mathrm{ml}$ samples was determined in parts-per-million (ppm) by a gas chromatograph (Quintron Microlyzer). All data were plotted against time and analysed by ANOVA (condition $v$ time, repeated measures). Individual orocaecal transit times were calculated based on the following criteria: A rise of at least $5 \mathrm{ppm}$ above baseline for at least two consecutive samples. Data were compared by paired t-test for differences between rest and stress condition. A 5\% level of significance was regarded as sufficient for statistical analysis.

EXPERIMENT 3

Eighteen female Wistar rats (mean weight: $196 \mathrm{~g}$ )

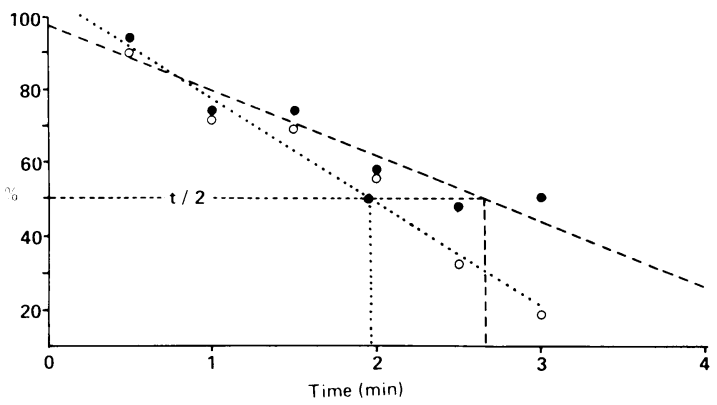

Fig. 1 Gastric emptying - \% of actual food intake of $5 \mathrm{~g}$ which remained in the stomach - after rest (dotted line) and after stress (dashed line). A linear regression provided best fit to the data; $t^{1 / 2}$ was calculated based on these functions: 1.97 h after rest and 2.66 h after stress. 
were housed individually and fed ad lib. Rats were controlled daily for their body weight.

A silicon catheter was implanted surgically into the caecum under light anaesthesia (Ketanest, $1.74 \mu \mathrm{l} / \mathrm{g}$ bw; Rompun, $0.65 \mu \mathrm{l} / \mathrm{g} \mathrm{bw}$ ). The proximal end of the catheter was sutured in the neck of the rat.

Three days after surgery $0.4 \mathrm{ml}$ of an iso-osmolar carmine red solution $(1.2 \mathrm{~g}$ in $100 \mathrm{ml}$ aqua dest) was infused into the caecum at $900 \mathrm{am}$. In five minute intervals the faecal output was controlled for discharge of coloured pellets while the rats remained in their home cages.

Seven days after surgery rats received an infusion of the carmine red marker at $900 \mathrm{am}$. The animals were then placed into the stress box described above. After flooding of the box with $500 \mathrm{ml}$ water at $30^{\circ} \mathrm{C}$, faecal output was monitored continuously for the occurrence of red coloured pellets for a maximum of six hours.

Colonic transit time was determined as the time interval between marker infusion and the discharge of the first red coloured faecal pellet. ${ }^{16}$ Differences between rest and stress condition were tested by paired $\mathrm{t}$-test for statistically significant differences. The level of significance was assessed to be $5 \%$.

\section{Results}

EXPERIMENT 1

All rats easily learned to eat up to $25 \mathrm{~g}$ of food within 30 minutes; after an initial drop of the mean body weight at days 7 to 5 the rats maintained their weight at the following days. At test day the weight was $177 \cdot 3(5 \cdot 9) \mathrm{g}$ (mean (SD)).

The average food uptake at test day was $5 \mathrm{~g}$ or $4 \mathrm{ml}$. Bivariate plots of the stomach content (\% of actual intake remaining in the stomach) against time showed that both under rest and under stress conditions the emptying curve followed a linear function:

$$
\begin{aligned}
& y=-28 \cdot 11 \times+105 \cdot 5 \text { for rest; } S D=26 \cdot 74, R M S Q=29 \cdot 49 \\
& y=-17 \cdot 94 \times+97 \cdot 7 \text { for stress; } S D=17 \cdot 64, R M S Q=36 \cdot 71 .
\end{aligned}
$$

$95 \cdot 8 \%$ and $88.2 \%$, respectively, of total variance of the data were explained by the curves which best fitted to the data. Average stomach emptying time $\left(t^{1} / 2\right)$ was 1.97 under rest and 2.66 under stress, the difference being statistically significant (Wilcoxon's signed-ranks test, $\mathrm{p}<0 \cdot 05$ ) (Fig. 1).

EXPERIMENT 2

The rats in experiment 2 also maintained their body weight after an initial drop.

Mean orocaecal transit under rest was $131 \cdot 4(20 \cdot 5)$ minutes. It significantly decreased after stress exposure to $86.3(11.4)$ minutes (paired t-test, $\mathrm{t}=2 \cdot 61, \mathrm{p}=0 \cdot(024)$. Mean breath hydrogen exhalation

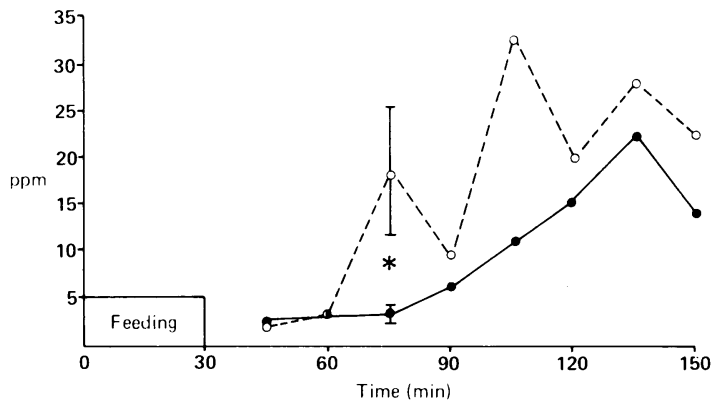

Fig. 2 Orocaecal transit time determined by cumulative breath hydrogen content of exhaled air after rest (solid line) and after stress (dashed line). A sustained rise of breath hydrogen above baseline of at least $5 \mathrm{ppm}$ for at least two consecutive samples indicates arrival of the bolus head in the caecum. Data were analysed by ANOVA for repeated measures; *indicates a significant diffpaired t-test.

for both conditions was statistically significant $(2 \times 5$ ANOVA, repeated measure, $\mathrm{F}=5 \cdot 87, \mathrm{p}=0 \cdot 0338$ ) (Fig. 2).

\section{EXPERIMENT 3}

The animals quickly recovered from surgery and maintained their body weight without substantial drop: at the first test day the average body weight was $196 \cdot 5(9 \cdot 4)$ g compared with $196 \cdot 2(9 \cdot 3)$ g presurgery (mean (SD)).

Mean colonic transit at rest was $15 \cdot 5(2 \cdot 0)$ hours (mean (SEM)). In no case a transit time of less than six hours was observed. Colonic transit time significantly $(\mathrm{p}<0.01)$ decreased from $15 \cdot 5(2 \cdot 01)$ hours at rest to $1.29(0.65)$ hours after stress. The mean number of pellets which were expelled within the first 30 minutes after marker application increased from zero to four per animal. Though the consistency of the faecal output could not be strictly assessed as the pellets fell into the water of the test box, the coloured pellets usually appeared to be soft or semiliquid.

\section{Discussion}

Previous investigations of the effects of experimental stress on gastrointestinal transit in rats have produced conflicting results. While in some studies an acceleration of gastric emptying or orocaecal transit following stress has been reported, ${ }^{125}$ in others a delay or no effect was observed. ${ }^{3+11}$ Similar differences have been reported with respect to colonic transit: most studies report an acceleration of colonic transit after stress, ${ }^{5 x-111}$ while others observed a delay or no effect. ${ }^{37}$ Faecal pellet output has been proposed as physiological marker of stress induced emotional arousal. ${ }^{\circ .}$ Differences in the experimental procedures may account for these divergent results. 
The experiments presented here were based on the following assumptions: (1) If the effect of an experimental stressor is studied, the experimental procedure should avoid to add further stressful experiences to the animal such as multiple handling or oral gavage of food. ${ }^{13}$ This can be achieved by training the animal to eat a standard volume of food within a given period. (2) The stressor should be of psychological nature and should minimise physical stress components (cold, heat, pain etc) which could produce the effects under investigation without mediation of the central nervous system. This was achieved by a "passive avoidance" ${ }^{\text {"s }}$ situation where the animal can avoid contact with the aversive physical event (water). (3) The marker technique used should be as physiological as possible; gastric emptying of solid food thus appears to be the more valid measure both under rest and stress than the emptying of a liquid bolus. ${ }^{1+}(4)$ The technique used should allow for multiple measurements of intestinal transit in individual animals to reduce between subject data variance. This can be achieved by noninvasive techniques like the breath hydrogen measurement ${ }^{17}$ or chronically implanted catheters.

Our data indicate that the stressor used in our studies exhibits differential effects in different parts of the gastrointestinal tract: It delays gastric emptying, acclerates orocaecal transit, and accelerates the colonic passage.

The opposite effects of stress in the upper gastrointestinal tract - namely, gastric emptying and orocaecal transit, which includes gastric emptying and small bowel transit - suggests independent control mechanisms for the stomach and small bowel transit. Such independence of stomach emptying and small bowel transit has been observed previously in animals" as well as in man. ${ }^{10}$ This may well account for conflicting data on stress effects reported in previous studies which usually determined gastric emptying and small bowel transit simultaneously by application of radio-isotope markers.

An alternative interpretation is stated by investigators who compared the effects of different stressors on the same part of the gastrointestinal tract and found stressor specific reactions of the system to account for differences reported in the literature. ${ }^{411}$ It needs to be clarified in further studies whether the acceleration of orocaecal transit after stress observed in our experiment is not effected by the delay in stomach emptying or whether the change in small bowel transit would have been even more pronounced than we could observe in our experiments.

It appears, instead, from our data that effects of a single stressor are more pronounced in the colon than the observed alteration in the upper gut. The observed changes in the colon appear to be similar to stress induced diarrhoea as occasionally reported in clinical observations in man. The acceleration observed exceeds that reported in previous studies. ${ }^{n}$ !2 In contrast with most stressors used in these studies, however, our model is thought to exert its effects through the central nervous system. Whether stress induced changes in colonic transit, however, may serve as a model for the 'irritable bowel syndrome' as stated recently" remains questionable, because the changes observed are of acute character rather than chronic changes, and the animals usually adapt to the stressor after several exposures..$^{13}$

Opioid and beta-adrenergic receptors have been reported to mediate stress effects on gastrointestinal

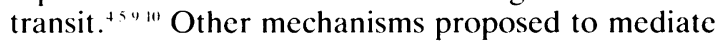
stress effects on the small and large intestine are peptides such as corticotrophin releasing factor (CRF). ${ }^{511021}$ Besides some agreement about the role of CRF being the 'master transmitter', however,"' mediating gastrointestinal stress effects at the central nervous site, data from these studies remain conflicting as both accelerations of gastric emptying induced by stress have been reported to be blocked by CRF as well as delays ${ }^{201}$ and acceleration of gastric emptying by $\mathrm{CRF}$ alone.

Part of these differences may also be the result of factors such as the sex of the animal ${ }^{22}$ or the oestrus cycle $^{33}$ which are known to effect gastrointestinal transit. While we controlled for the former, the latter has not been taken into consideration in all studies conducted so far. It is suggested, however, by the study reported here that a psychological stress model which allows for multiple measurement under physiological conditions is a prerequisite for further investigations of mediators for stress effects on gastrointestinal transit.

Supported by grants En 50/2 and Wi $285 / 5$ from the Deutsche Forschungsgemeinschaft .

\section{References}

1 Brown MS, Groves WG. Intestinal propulsion in restrained and unrestrained rats. Proc Soc Exp Biol Med 1966; 121: 989-95.

2 Glavin GB, Mikhail AA. Stress duration, gastric emptying, and ulcer development in three strains of rats. Biochem Med 1977: 18: 58-63.

3 Galligan JJ, Porreca F, Burks TF. Footshock produces analgesia but no gastrointestinal motility effects in the rat. Life Sci 1983; 33: 473-5.

4 Appelbaum BD. Hotzman SG. Restraint stress has no effect on morphine-induced inhibition of gastrointestinal transit in the rat. Physiol Behav 1985; 34: 995-7.

5 Gue M. Fioramont Bueno L. Disturbance of gastric emptying and intestinal transit induced by acoustic and cold stress in mice. Am J Physiol 1987; 253: G124-8. 
6 Bindra D. Thompson WR. An evaluation of defecation and urination as measures of fearfulness. J Comp Physiol Psychol 1953; 46: 43-5.

7 Roth KA. Katz RJ. Stress, behavioral arousal, and open field activity - a reexamination of emotionality in the rat. Neurosci Biobehav Rev 1979; 3: 247-63.

8 Ossenkopp KP. Frisken NL. Defaecation as an index of motion sickness in the rat. Physiol Psychol 1982; 10: 355-60).

9 Williams CL, Villar RG, Peterson JM, Burks TF. Stressinduced changes in intestinal transit in the rat: A model for irritable bowel syndrome. Gastroenterology 1987; 94: $611-21$.

10 Williams CL, Peterson JM, Villar RG, Burks TF. Corticotrophin-releasing factor directly mediates colonic responses to stress. Am J Physiol 1987; 253: G582-6.

11 Barone FC, Deegan JF, Fowler FJ, Fondacaro JD, Ornsbee III HS. A model of stress-induced increased faccal pellet output and colonic transit [Abstract]. Gastroenterology 1986; 90: 1137.

12 Green AF. Comparative effects of analgesics on pain threshold, respiratory frequency and gastrointestinal propulsion. Br J Pharmacol 1959; 14: 26-34.

13 Kvetnansky R. Sun CL, Lake CR, Thoa N, Torda T, Kopin IP. Effects of handling and forced immobilazation on rat plasma levels of epinephrine, norepinephrinc, and dopamine- $\beta$-hydroxylase. Endocrinology 1978; 103: 1868-74.

14 Meyer HH. Motility of the stomach and gastroduodenal junction. In: Johnson LJ, ed. Physiology of the gastro- intestinal tract. Vol. 1. New York: Raven Press, 1987: 61.3-29.

15 Mowrer OH. Learning theory and behavior. New York: Wilcy, 1960.

16 Elashoff JD, Reedy TJ, Meyer JH. Analysis of gastric emptying data. Gastroenterology 1982; 83: 13()6-12.

17 Brown NJ, Rumsey RDE, Read NW. Adaptation of hydrogen analysis to measure stomach to caccum transit time in the rat. Gut 1987; 28: 849-54.

18 Leng-Peschlow E. Acceleration of large intestine transit time in rats by sennosides and related compounds. J Pharm Pharmacol 1986: 38: 369-73.

19 Read NW, Cammack J, Edwards C. Holgate AM, Cann PA, Brown C. Is the transit time of a meal through the small intestine related to the rate at which it leaves the stomach? Gut 1982; 23: 824-8.

20 Lenz HJ, Raedler A, Greten H, Brown MR. CRF initiates biological actions within the brain that are observed in response to stress. Am J Physiol 1987; 252: 34-9.

21 Taché Y, Macda-Hagiwara M, Turkelson CM. Central nervous system action of corticotrophin-releasing factor to inhibit gastric emptying in rats. Am J Physiol 1987; 253: G24l-5.

22 Hutson W, Roehrkasse R, Kaslewicz F, Stoney B, Wald A. Influence of sex and weight on gastric emptying (GE) and antral motility (AM). [Abstract]. Gastroenterology 1987; 92: 1443.

23 Scott LD, Lester R, Van Thiel D, Wald A. Pregnancyrelated changes in small intestinal myoclectrical activity in the rat. Gastroenterology $1983 ; 84: 301-5$. 